\title{
MÁS TRÁNSITO DE HIDROCARBUROS EN NUESTRO MAR: ponen en peligro nuestros alimentos de origen marino
}

\author{
MORE TRANSIT OF HYDROCARBONS IN OUR SEA: they \\ endanger our foods of marine origin
}

Manuel S. Quispe-Villanueva

Universidad Nacional del Santa. Ancash, Perú

Recibido: 23/11/2021 Revisado: 17/12/2021_ Aceptado: 21/12/2021 Publicado: 15/01/2022

\section{RESUMEN}

Actualmente nos estamos dirigiendo hacia un cambio de la fuente de energía del petróleo y sus derivados hacia el uso de energía eólica, solar, hidrógeno, hidroeléctricas, entre otras. Sin embargo, nuestro país continúa otorgando permisos para la exploración y explotación de hidrocarburos en nuestro mar. Por tal motivo el propósito de la presente revisión es proporcionar evidencia científica para generar el mejor criterio de opinión en la población respecto a la licencia otorgada por el Ministerio de energía y minas a las empresas Perupetro y Tullow Perú Limited para la exploración y perforación de hidrocarburos frente a la región Ancash en los lotes Z-67 y Z-68. Se realizó la búsqueda en el Medline (PubMed), Web of Science, repositorios de las universidades y la biblioteca del Concytec. Para todos los casos se han utilizado las palabras clave: derrame de hidrocarburos o petróleo. Se llegó a las siguientes conclusiones: Las escasas investigaciones respecto al impacto de los hidrocarburos en nuestro mar peruano impiden tener claro sus efectos en el ambiente marino y en sus recursos hidrobiológicos. Y el aumento del tránsito de embarcaciones con hidrocarburos en nuestro mar, hace que aumenten las probabilidades de derrame de los mismos en nuestro mar poniendo en peligro nuestros alimentos de origen marino.

Palabras clave: hidrocarburos; derrame de petróleo.

\section{ABSTRACT}

We are currently heading towards a change in the energy source of oil and its derivatives towards the use of wind, solar, hydrogen and hydroelectric energy, among others. However, our country continues to grant permits for the exploration and exploitation of hydrocarbons in our sea. For this reason, the purpose of this review is to provide scientific evidence to generate the best opinion criteria in the population regarding the license granted by the Ministry of Energy and Mines to the companies Perupetro and Tullow Peru Limited for the exploration and drilling of hydrocarbons. in front of the Ancash region in lots Z-67 and Z-68. The search was carried out in the Medline (PubMed), Web of Science, university repositories and the Concytec 
library. For all cases, the keywords have been used: oil or hydrocarbon spill. The following conclusions were reached: The few investigations regarding the impact of hydrocarbons in our Peruvian sea make it difficult to be clear about their effects on the marine environment and on its hydrobiological resources. And the increase in the transit of vessels with hydrocarbons in our sea increases the chances of oil spills in our sea, endangering our food of marine origin. Keywords: Hydrocarbons; oil spill.

\section{INTRODUCCIÓN}

El deterioro avanzado del mar peruano es ocasionado por diferentes fuentes de contaminación como la minería, la agricultura, fábricas, desagües de las ciudades aledañas, los hidrocarburos procedentes de las plataformas petroleras y los derrames de petróleo ocasionado por el transporte del hidrocarburo. El alto tránsito de buques petroleros que tiene la región de Pisco, se va a incrementar cuando concluyan las obras en el Puerto de Chancay que está construyendo la naviera china Cosco Shipping en convenio con la minera peruana Volcán y que entrará en vigencia en 2022, y con esto se aumentan los riesgos de contaminación por derrame de petróleo. Si continúan las construcciones de plataformas de hidrocarburos en nuestra región marina y a la vez, la minería sigue contaminando nuestros ríos, es de esperar que nuestros recursos hidrobiológicos, en general, dejarán de ser comestibles debido a la acumulación de metales pesados y de hidrocarburos, afectándose de esta manera el consumo de pescado y la pesquería. El propósito de la presente revisión es proporcionar evidencia científica para generar el mejor criterio de opinión en la población respecto a la licencia otorgada por el Ministerio de energía y minas a las empresas Perupetro y Tullow Perú Limited para la exploración y perforación de hidrocarburos frente a la región Ancash en los lotes Z-67 y Z-68.

\section{MATERIALES Y MÉTODOS}

Se ha realizado la búsqueda electrónica sin restricción de fecha e idioma en el Medline (PubMed), Web of Science, repositorios de las universidades y la biblioteca del Concytec. Para todos los casos se han utilizado las palabras clave: derrame de hidrocarburos o petróleo.

\section{RESULTADOS Y DISCUSIÓN}

\section{Los derrames de petróleo}

Nigeria, entre 1976 y 1991, reporta un total de 2976 derrames, haciendo un volumen de 2 millones de barriles de petróleo crudo, vertidos en el medio ambiente y con daños para la salud. Afecto, particularmente las comunidades costeras del sur de dicho país. El impacto ambiental y sanitario de estos derrames se ve agravado por los procesos y políticas inadecuados de evaluación del impacto ambiental, sanitario y particularmente en el ámbito de la participación pública y el control gubernamental de los proyectos petroleros (Edoigiawerie y Spickett, 1995).

Desde el inicio de la perforación en alta mar, los residuos han recibido mucha atención en la industria del petróleo y el gas. Sin embargo, el conocimiento del impacto ambiental marino asociado a la eliminación de dichos residuos, aún es limitado (Durrieu, Galgani, Jorissen y Cazes, 2007). 
La industrialización de las profundidades marinas se está expandiendo en todo el mundo. El aumento de las actividades de exploración de petróleo y gas en ausencia de suficientes datos de referencia en los ecosistemas de aguas profundas ha dificultado la gestión ambiental. El impacto ambiental puede persistir en las profundidades del mar durante muchos años y probablemente más para los ecosistemas frágiles, como los corales de agua fría. La implementación de estrategias de gestión debe considerar zonas de amortiguamiento mínimas para desplazar la actividad industrial más allá del rango de impactos típicos: al menos $2 \mathrm{~km}$ de cualquier punto de descarga e infraestructura de superficie y $200 \mathrm{~m}$ de la infraestructura del lecho marino sin descargas esperadas. Aunque la gestión de los recursos naturales es, posiblemente, más desafiante en entornos de aguas profundas, la inclusión de estas herramientas de conservación comprobadas contribuye a estrategias sólidas de gestión ambiental para la extracción de petróleo y gas en las profundidades marinas (Cordones et al 2016).

Es evidente las emisiones de metano, petróleo y gas de las plataformas en alta mar en el sudeste asiático (Nara et al., 2014). Todas las plataformas de petróleo y gas observadas tienen fugas de $\mathrm{CH} 4$ durante el funcionamiento normal, y gran parte de esta fuga no se ha incluido en los inventarios de emisiones de las plataformas petroleras en el Reino Unido, y se sugiere que se realicen mediciones adicionales de las operaciones en la plataforma de producción de petróleo y gas en alta mar (por ejemplo, Arabia Saudita, Brasil, México, Noruega y Estados Unidos) para informar adecuademente las estimaciones de fugas, y que estas mediciones se utilicen para mejorar los inventarios de emisiones de metano en el mundo (Riddick et al., 2019).

Las plataformas petroleras emiten grandes cantidades de metano y otros gases que son mal cuantificadas, y los estudios de inventarios en tierra muestran subestimaciones sistemáticas. Atribuimos este desacuerdo a recuentos incompletos y factores que subestiman las emisiones de las plataformas de aguas poco profundas (Gorchov, Kort, Conley y Smith, 2020).

Los grandes derrames de petróleo son eventos catastróficos que afectan enormemente al medio ambiente y la sociedad, pero determinar su extensión espacial es una tarea muy compleja. Durante la explosión de Deepwater Horizon aproximadamente 149,000 km2 del Golfo de México fueron cubiertos por mareas negras, que se extendió más allá de los límites del satélite y vastas áreas fueron cerradas para la pesca por exposición tóxica al petróleo. El aumento global de las actividades relacionadas con la producción de petróleo, hace necesaria una evaluación cuidadosa de la extensión total de los derrames de petróleo para maximizar la seguridad ambiental y pública (Berenshtein et al., 2020).

\section{La causa de los derrames de petróleo}

El transporte marítimo de petróleo trae enormes beneficios económicos a muchos países; por otro lado, también trae enormes amenazas contra la salud, la vida, la propiedad y el medio ambiente. Se ha reportado que la contaminación por derrames de petróleo entre 1970 y 2017 ha sido causada por el transporte, evidenciado porque existe una dependencia significativa entre la cantidad de accidentes por derrames de petróleo y la cantidad de productos petroleros transportados (Galieriková y Materna, 2020). 
El atentado contra la salud de los seres vivos

Los accidentes de derrames de petróleo afectan drásticamente el medio ambiente y el ecosistema en las zonas intermareales. El aceite derramado penetra en los sedimentos y se acumula causando efectos letales o subletales en los invertebrados bentónicos. Las estructuras poblacionales de la macrofauna se alteran drásticamente en cuanto al número, abundancia y biomasa de las especies. En general, se necesita mucho tiempo para que la macrofauna se recupere después de un derrame de petróleo de alta concentración, que en un derrame de baja concentración (Zhou, et al., 2019).

El 2010, la Costa del Golfo de México experimentó el mayor derrame de petróleo de toda su historia. La evaporación del petróleo crudo y los agentes químicos dispersantes aumentaron las concentraciones de materia particulada 2,5, óxido de nitrógeno, dióxido de azufre y monóxido de carbono en los condados costeros afectados, causando aumento de la incidencia de bajo peso al nacer $(<2500$ g) y de recién nacidos prematuros $(<37$ semanas de gestación). Los efectos de heterogeneidad revelan resultados de salud infantil adversos más pronunciados para las madres de las etnias afroamericana e hispanas, menos educadas, solteras y más jóvenes (Beland y Oloomi 2019). Además, se reporta que existe asociación entre la exposición al derrame de petróleo crudo y los síntomas neurológicos agudos (Krishnamurthy et al., 2019).

Desde el punto de vista de la salud pública, la explotación del recurso petróleo representa una amenaza por el fracaso fundamental de las teorías de desarrollo dominantes, más que el retraso en la creación de un entorno económico y de gobernanza adecuado para el progreso social (Calain, 2008).

\section{La contaminación del litoral peruano}

El primer pozo petrolero perforado en el Perú, y en Sudamérica, fue en 1863 en la ciudad de Zorritos, (al cual debe su nombre por los olores fétidos que emitía) Tumbes. Se han explorado tanto las cuencas costeras continentales como la plataforma continental marina. En los primeros días de la exploración, el énfasis se centró en la zona continental al norte del país, pero los avances en la comprensión de los conceptos geológicos indican que todavía existen sistemas petroleros sin explorar en la mayoría de las cuencas costa afuera ubicadas más allá de la isóbata de 120 metros que había sido el límite para la exploración, pero que son accesibles utilizando la tecnología de exploración actual. En la mayoría de las cuencas, se evidencian tanto en el fondo como en la superficie marina, la presencia de emanaciones líquidas y gaseosas de hidrocarburos, denotando así la existencia de sistemas petroleros activos en sus profundidades (Bolaños, 2017).

El litoral peruano presenta hidrocarburos de petróleo cuyas concentraciones están muy influenciadas por la actividad petrolera, como es el caso de la Bahía del Callao $(3,74$ y 1,12 ug/L), Pisco (0,96 y 0,68ug/L), Sechura $(0,73$ y $0,79 \mathrm{ug} / \mathrm{L})$ y Talara $(0,73 \mathrm{ug} / \mathrm{L})$, en esta última se encuentra la Refinería de Talara y también la actividad en pozos petroleros. Respecto a los derrames de petróleo, Pisco, tiene un alto tránsito de buques petroleros y que ya han ocasionado derrame afectando las playas adyacentes. Además, el incremento de la actividad petrolera, de exploración y explotación de yacimientos en la plataforma continental, está incrementando el riesgo de contamina- 
ción por petróleo crudo. Finalmente, la minería de la región Ancash contamina sus ríos con metales pesados y de manera puntual también lo hacen en los sedimentos de la bahía de Chimbote (Sánchez, Blas y Chau, 2010).

Hooker y Pestana (2012), indican que varios países del mundo demuestran la importancia de las plataformas petroleras y gasíferas como arrecifes artificiales, en ella se concentra una importante biodiversidad marina que incluye especies de importancia comercial.

El Perú está incrementando la instalación de plataformas de producción de hidrocarburos en el mar, sin embargo, se desconoce el impacto de estas estructuras en la biota submarina.

\section{Medidas urgentes a tomar}

1. Tenemos la emergencia de determinar la concentración actual de hidrocarburos aromáticos policíclicos alquilados presente en invertebrados comestibles como los mariscos. Farrington (2020), afirma que es necesario actualizar los protocolos de evaluación de riesgos para la salud humana en lo que respecta a los hidrocarburos aromáticos policíclicos en los mariscos, y que estos deberían incluirse en la evaluación de riesgos para la salud humana y no ser descartados cuando presenten niveles muy bajos.

2. Desde la perspectiva de salud ambiental, es importante investigar cómo influye la extracción no convencional de petróleo y gas en los comportamientos y prácticas sociales de la población, utilizando la investigación etnográfica y longitudinal (Cotton y Parry, 2018).

3. Es necesario desarrollar modelos que re- flejen las distribuciones de concentración de derrames de hidrocarburos dada la influencia de factores ambientales, físicos, biológicos y químicos. Además, las actividades humanas deben cuantificarse para diferentes poblaciones, incluidos los trabajadores de respuesta a emergencias, los pescadores, los consumidores de mariscos y los niños que juegan en las playas que pueden verse afectadas por los derrames de petróleo. También es necesario trabajar en el desarrollo de perfiles toxicológicos completos para la mayoría de los productos químicos, incluidos los dispersantes que se utilizan en los derrames de petróleo para estimar la toxicidad de las mezclas (Ferguson, Solo y Mena, 2020).

4. La información recogida de las declaraciones del impacto ambiental en una petrolera debe depender del contexto, los desafíos de revisar estas declaraciones no están en su extensión sino en que se derivan de información irrelevante, así como de una capacidad débil para procesar información. Por lo tanto, se debe mejorar el alcance de la evaluación y fortalecer la capacidad en la toma de decisiones (Fonseca y Rivera, 2020).

\section{CONCLUSIONES}

- Las escasas investigaciones respecto al impacto de los hidrocarburos en el mar peruano impiden tener claro sus efectos en el ambiente marino y en sus recursos hidrobiológicos.

El aumento del tránsito de embarcaciones con hidrocarburos en el mar peruano, hace que aumenten las probabilidades de derrame poniendo en peligro los alimentos de origen marino. 


\section{REFERENCIAS BIBLIOGRÁFICAS}

Beland L.P., Oloomi S. (2019). Environmental disaster, pollution and infant health: Evidence from the Deepwater Horizon oil spill. Journal of Environmental Economics and Management, 2019. Volume 98, November 2019, 102265. https://doi.org/10.1016/j.jeem.2019.102265

Berenshtein, I., Paris, C. B., Perlin, N., Alloy, M. M., Joye, S. B., \& Murawski, S. (2020). Invisible oil beyond the Deepwater Horizon satellite footprint. Science Advances, 6(7), [eaaw8863]. https://doi.org/10.1126/sciadv.aaw8863

Bolaños Z.R (2017). Reseña Histórica de la Exploración por Petróleo en las Cuencas Costeras del Perú. Boletín de la Sociedad Geológica del Perú. v. 112, p. 001-013

Calain P. (2008). Oil for health in sub-Saharan Africa: health systems in a 'resource curse' environment. Globalization and health, 4, 10. https://doi.org/10.1186/1744-8603-4-10

Chen, J., Zhang, W., Wan, Z., Li, S., Huang, T., \& Fei, Y. (2019). Oil spills from global tankers: Status review and future governance. Journal of Cleaner Production, 227, 20-32. https://doi.org/10.1016/j.trpro.2020.02.039

Cordes EE, Jones DOB, Schlacher TA, Amon DJ, Bernardino AF, Brooke S, Carney R, DeLeo DM, Dunlop KM, Escobar-Briones EG, Gates AR, Génio L, Gobin J, Henry L-A, Herrera S, Hoyt S, Joye M, Kark S, Mestre NC, Metaxas A, Pfeifer S, Sink K, Sweetman AK and Witte U (2016) Environmental Impacts of the Deep-Water Oil and Gas Industry: A Review to Guide Management Strategies. Front. Environ. Sci. 4:58. https://doi.org/10.3389/fenvs.2016.00058

Cotton I.M. \& Parry C. (2018). Beyond opposition and acceptance: Examining public perceptions of the environmental and health impacts of unconventional oil and gas extraction. Current Opinion in Environmental Science \& Health. Volume 3, Pages 8-13 https://doi.org/10.1016/j.coesh.2018.01.001

Durrieu, J., Galgani, F., Jorissen, F., \& Cazes, L. (2007). Quantitative Assessment Of The Environmental Impact Of Offshore Drilling Activities Using Benthic Fauna: Lessons Learnt From Actual Cases. Offshore Mediterranean Conference. https://www.onepetro.org/conference-paper/OMC-2007-103

Edoigiawerie, C., \& Spickett, J. (1995). The environmental impact of petroleum on the environment. African journal of health sciences, 2(2), 269-276. https://pubmed.ncbi.nlm.nih.gov/12160434/ 
Ferguson, A., Solo-Gabriele, H., \& Mena, K. (2020). Assessment for oil spill chemicals: Current knowledge, data gaps, and uncertainties addressing human physical health risk. Marine pollution bulletin, 150, 110746. https://doi.org/10.1016/j.marpolbul.2019.110746

Fonseca, A., \& Rivera F. G. M. (2020). Reviewers' perceptions of the volume of information provided in environmental impact statements: The case for refocusing attention on what is relevant. Journal of cleaner production, 251, https://doi.org/10.1016/j.jclepro.2019.119757

Galieriková, A., \& Materna, M. (2020). World Seaborne Trade with Oil: One of Main Cause for Oil Spills? Transportation research procedia, 44, 297-304. https://doi.org/10.1016/j.trpro.2020.02.039

Gorchov N.A., Kort E., Conley, S.A. y Smith, M. L. (2020). Airborne Assessment of Methane Emissions from Offshore Platforms in the U.S. Gulf of Mexico. Environ. Sci. Technol. 2020, 54, 8, 5112-5120. https://doi.org/10.1021/acs.est.0c00179.

Krishnamurthy, J., Engel, L. S., Wang, L., Schwartz, E. G., Christenbury, K., Kondrup, B., Barrett, J., \& Rusiecki, J. A. (2019). Neurological symptoms associated with oil spill response exposures: Results from the Deepwater Horizon Oil Spill Coast Guard Cohort Study. Environment international, 131, 104963. https://doi.org/10.1016/j.envint.2019.104963

Nara, H., Tanimoto, H., Tohjima, Mukai H., Yukihiro N., Machida T. 2014. Emissions of methane from offshore oil and gas platforms in Southeast Asia. Sci Rep 4, 6503 (2014). https://doi.org/10.1038/srep06503

Riddick, S. N., Mauzerall, D. L., Celia, M., Harris, N. R. P., Allen, G., Pitt, J., Staunton-Sykes, J., Forster, G. L., Kang, M., Lowry, D., Nisbet, E. G., and Manning, A. J. (2019). Methane emissions from oil and gas platforms in the North Sea, Atmos. Chem. Phys., 19, $9787-$ 9796, https://doi.org/10.5194/acp-19-9787-2019

Sánchez R. G., Blas L.N. \& Chau F. G. (2010) Informe sobre el Estado del Medio Ambiente marino del Perú. Ministerio de la Producción e Instituto del mar del Perú. http://cpps.dyndns.info/cpps-docs-web/planaccion/docs2010/oct/XVII_AG_GC/18.Contaminacion.marina.Informe.final.Peru.pdf

Zhou, Z., Li, X., Chen, L., Li, B., Wang, C., Guo, J., Shi, P., Yang, L., Liu, B., \& Song, B. (2019). Effects of diesel oil spill on macrobenthic assemblages at the intertidal zone: A mesocosm experiment in situ. Marine environmental research, 152, 104823. https://doi.org/10.1016/j.marenvres.2019.104823 